\title{
Streaming Trend Detection in Twitter
}

\author{
James Benhardus
}

\begin{abstract}
Twitter is a popular microblogging and social networking service with over 100 million users. Users create short messages pertaining to a wide variety of topics. Certain topics are highlighted by Twitter as the most popular and are known as "trending topics." In this paper, we will outline methodologies of detecting and identifying trending topics from streaming data. Data from Twitter's streaming API will be collected and put into documents of equal duration. Data collection procedures will allow for analysis over multiple timespans, including those not currently associated with Twitter-identified trending topics. Term frequency-inverse document frequency analysis and relative normalized term frequency analysis are performed on the documents to identify the trending topics. Relative normalized term frequency analysis identifies unigrams, bigrams, and trigrams as trending topics, while term frequeny-inverse document frequency analysis identifies unigrams as trending topics.
\end{abstract}

Index Terms-microblogs, trend detection, natural language processing

\section{INTRODUCTION}

$\mathbf{T}$ WITTER is a popular microblogging and social networking service that presents many opportunities for research in natural language processing (NLP) and machine learning. Since its inception in 2006, Twitter has grown to the point where http://twitter.com is the 11 th most visited website in the world, and the 8th most visited site in the United States ${ }^{1}$, and over 100 million Twitter accounts have been created ${ }^{2}$. Users of Twitter post short (less than than or equal to 140 character) messages, called "tweets," on a variety of topics, ranging from news events and pop culture, to mundane daily events and spam postings. As of February 2010, users of Twitter were producing 50 million tweets per day, an average of 600 tweets per second ${ }^{3}$.

Twitter presents some intriguing opportunites for applications of NLP and machine learning. One such aspect of Twitter that provides opportunities is trending topics - words and phrases, highlighted on the main page of Twitter, that are currently popular in users' tweets. Trending topics are identified for the past hour, day and week. Examples of trending topics can be seen in Fig. 1 and Fig. 2. Trending topics are supposed to represent the popular "topics of conversation," so to speak, among the users of Twitter. Determining trending topics can be considered a type of First Story Detection (FSD), a subset of the larger problem known as Topic Detection and

J. Benhardus (Physics Department - Bethel University, St. Paul, MN 55112) is a rising senior participating in a Summer 2010 National Science Foundation REU for Artificial Intelligence, Natural Language Processing and Information Retrieval at the University of Colorado at Colorado Springs, Colorado Springs, CO, 80918.

email: benjam@bethel.edu

${ }^{1}$ http://www.alexa.com/siteinfo/twitter.com

${ }^{2} \mathrm{http}$ ///economictimes.indiatimes.com/infotech/internet/Twitter-snags-over100-million-users-eyes-money-making/articleshow/5808927.cms

${ }^{3}$ http://blog.twitter.com/2010/02/measuring-tweets.html
Tracking (TDT) [1]. The popularity and growth of Twitter presents some challenges for applications of NLP and machine learning, however. The length restrictions of the messages create syntactical and structural conventions that are not seen in more traditional corpora, and the size of the Twitter network produces a continuously changing, dynamic corpus. In addition, there is quite a lot of content on Twitter that would be classified as unimportant to an outside observer, consisting of personal information or spam, which must be filtered out in order to accurately identify the elements of the corpus that are relevant to the Twitter community as a whole, and could thus be considered to be potential trending topics. The challenge of Twitter's popularity is that in order to detect and identify trending topics, one must sample and analyze a large volume of streaming data. This paper will propose methods of using natural language processing techniques on streaming data from Twitter to identify trending topics.

\section{RELATED WORK}

While there is a large body of work pertaining to natural language processing, applying NLP techniques to Twitter is a fairly recent development, due in part to the fact that Twitter has only been in existence since $2006^{4}$. In this relatively short span of time, however, there have been many insightful analyses of Twitter. In particular, there are several recent applications natural language processing techniques to Twitter:

- Twitter has been used to study the dynamics of social networks, particularly the temporal behavior of social networks [11], or the behavior of social neworks during disasters, such as earthquakes [8], [14].

- First story detection has been applied to Twitter to identify the first tweets to mention a particular event [12].

- Data mining from trending topics have also been applied to Twitter to summarize trending topics [17] and to analyze how trending topics change over time [2].

- In addition to applications of NLP techniques to Twitter, trend and event detection techniques have also been applied to other online entities such as weblogs [3], [4], news stories [1], [10], [20], or scientific journal collections [16], [19].

\section{Problem Definition}

The main goal of this project is to detect and identify trending topics from streaming Twitter data. To accurately define the problem, the first step must be to define explicitly what constitutes a trending topic. In [4], topics are defined as consisting of a combination of chatter, which is characterized by persistent discussion at a constant level and is largely

\footnotetext{
${ }^{4}$ http://en.wikipedia.org/wiki/Twitter
} 
user-initiated, and spikes, which are characterized by shortterm, high intensity discussion that is often in response to a recent event. In general, trending topics consist mainly of spikes. However, trending topics can also consist of a fairly even combination of spikes and chatter, or of mainly chatter. Examples from Fig. 1 and Fig. 2 of trending topics that could be considered to consist mainly of spikes are:

- CAlA BOCA GALVAO

- Gonzalo Higuain

- Sani Kaita

- FIFA World Cup

- \#worldcup

- Grecia

- Maradona

- Vuvuzela

- Oil Spill

- Tony Hayward

- Oswalt

- Shirley Sherrod

- Breitbart

Examples from Fig. 1 and Fig. 2 of trending topics that could be considered to be a fairly even combination of spikes and chatter are:

- \#theview

- Jersey Shore tonight

- Thor

Examples from Fig. 1 and Fig. 2 of trending topics that could be considered too consist mainly of chatter are:

- Inception

- \#iconfess

- \#dontcountonit

- BUSTIN DREW JIEBER

The "\#" symbol at the beginning of "\#worldcup", "\#iconfess", "\#theview", and "\#dontcountonit" is called a "hashtag," and is used by Twitter users to classify their tweets as pertaining to a particular topic. In addition to spikes and chatter, a trending topic can also be the result of advertisement, as is the case for the final trending topic in Fig. 1, "Toy Story 3." In this third

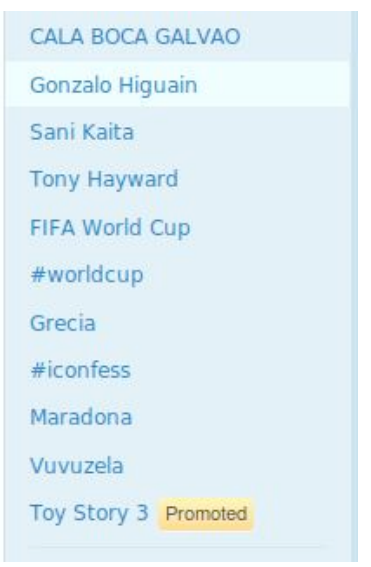

Fig. 1: A list of trending topics as identified by Twitter from 17 June 2010.

(Source: http://twitter.com) possibility, the trending topic is associated with a "Promoted" tweet - a hybrid tweet-advertisement which is displayed at the top of search results on relevant topics ${ }^{5}$.

While the classification of a trending topic as consisting of spikes or chatter is helpful for the understanding of the nature of trending topics, it is not directly useful in the identification or classification of terms as trending topics. Our working definition of a trending topic shall be a word or phrase that is experiencing an increase in usage, both in relation to its long-term usage and in relation to the usage of other words. More techincal definitions of trending topics shall be used in the actual experiments, and shall be described in the "Methodologies" section.

In addition to defining what constitutes a trending topic, we must also define what constitutes success for a particular methodology. As the goal of the project is to develop a method of identifying trending topics that is independent of the method used by Twitter, simple agreement with the Twitter-identified trending topics is both unambitious and potentially unrealistic without replicating Twitter's methodology, which happens to be proprietary. As such, we shall define a successful method as a method that produces relevant topics at a rate of at least $75 \%$ of the rate returned by Twitter's method, with an agreement of at least $50 \%$ with the terms produced by Twitter's method. The details of computing relevance and agreement shall be discussed in the "Evaluation Measures" section.

\section{Methodologies}

Multiple methodologies were implemented, making use of one or more selection criteria. Each selection criterion will be discussed in its own subsection. All methods implemented made use both of the Twitter Streaming $\mathrm{API}^{6}$ and the Edinburgh Twitter corpus [13], a collection of approximately 97 million tweets collected between November 2009 and February 2010. The Edinburgh Twitter corpus was used to provide baseline measurement against the data from the Twitter Streaming API. For each source, tweets were temporally

\footnotetext{
${ }^{5}$ http://blog.twitter.com/2010/04/hello-world.html

${ }^{6} \mathrm{http} / / /$ stream.twitter.com/1/statuses/sample.json (see documentation at http://apiwiki.twitter.com/Streaming-API-Documentation)
}

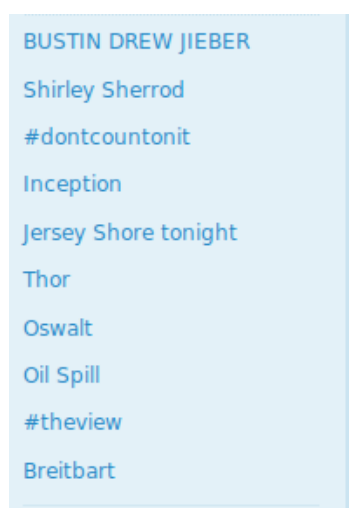

Fig. 2: A list of trending topics as identified by Twitter from 29 July 2010.

(Source: http://twitter.com) 
grouped into "bag of words" style collections, or "documents." These documents were be normalized by duration, meaning that each document corresponds to the tweets posted in a certain constant length of time. The Edinburgh Twitter corpus was divided into 1212 sections, each consisting of one hour's worth of tweets. The tweets from the Twitter Streaming API were grouped into sections corresponding to either ten minutes, fifteen minutes, or one hour's worth of data collection.

\section{A. Frequency}

The first criterion used was simply the raw frequency of each term. This criterion was used mainly as a threshold criterion, to be used with one or more of the other criteria. Using raw frequency by itself has major drawbacks, as the most frequent terms in the stream are the terms that carry the least information, such as "the", "and", or "rt" (an abbreviation for "retweet," a term used when one Tiwtter user reposts another Twitter user's tweet). The majority of the most common words can be classified as stop words, and filtered out of the stream. Generation of a stop word list shall be discussed in the "Experiments" section.

\section{B. $T F-I D F$}

The second criterion implemented involved analyzing each document using an application of tf-idf weighting. Tf-idf weighting is a information retrevial technique that weights a document's relevance to a query based on a composite of the query's term frequency and inverse document frequency [15]. Term frequency can be defined as either

$$
t f_{i, j}=n_{i, j}
$$

or

$$
t f_{i, j}=\frac{n_{i, j}}{N}
$$

where $n_{i, j}$ is the number of times word $i$ occurs in document $j$ and

$$
N=\sum_{k} n_{k, j}
$$

is the total number of words in document $j$. The second definition of $t f_{i, j}$ is often referred to as the normalized term frequency. Inverse document frequency is defined as

$$
i d f_{i}=\log \left(\frac{D}{d_{i}}\right)
$$

where $d_{i}$ is the number of documents that contain word $i$ and $D$ is the total number of documents. Put simply, the weight of a document will be higher if the number of times a word occurs in a document is higher, or if the number of documents containing that word is lower; similarly, the weight of a document will be lower if the number of times a word occurs in a document is lower, or if the number of documents containing that word is higher [5].

\section{Normalized Term Frequency}

The third criterion implemented involved utilizing only the term frequency of each element, rather than both the term frequency and the inverse document frequency. For this method, a normalized term frequency was used, defined as

$$
t f_{\text {norm }_{i, j}}=\frac{n_{i, j}}{\sum_{k} n_{k, j}} * 10^{6}
$$

where $n_{i}$ is the number of times word $i$ occurs in document $j$ and $\sum_{k} n_{k, j}$ is the total number of words in document $j$. Due to the large number of words found in the documents, a scaling factor of $10^{6}$ was used, meaning $t f_{\text {norm }_{i, j}}$ can be thought in terms of frequency per million words. Each word in the test document was given a trending score, defined as

$$
t s_{i, j}=\frac{t f_{\text {norm }_{i, j}}}{a t f_{\text {norm }_{i, S}}}
$$

in which

$$
\text { atf }_{\text {norm } i, S}=\sum_{S=\left\{s_{1}, \ldots, s_{p}\right\}} \frac{t f_{\text {norm }_{i, s_{k}}}}{p}
$$

where $S$ is the set of $p$ baseline documents to which the test document was compared.

\section{Entropy}

The fourth criterion implemented was entropy. To calculate the entropy of a term, all of the tweets containing that term are collected. As it is used in this project, the entropy of a term $i$ is defined as

$$
H_{i}=-\sum_{j} \frac{n_{j, i}}{N} \log \left(\frac{n_{j, i}}{N}\right)
$$

where $n_{j, i}$ is the number of times word $j$ occurs in the collection of tweets containing term $i$ and

$$
N=\sum_{j} n_{j, i}
$$

is the total number of words in the collection of tweets containing term $i$. Entropy proved to be a helpful parameter to use in filtering out terms that could be classified as spam.

\section{EXPERIMENTS}

Two experiments were run, implementing slightly different methodologies, but following the same general format. Unless stated otherwise, the process described was used for both experiments.

\section{A. Data Collection}

Data was collected using the Twitter streaming API, with the gardenhose tweet stream providing the input data and the trends/location stream providing the list of terms identified by Twitter as trending topics. The gardenhose streaming API is a limited stream that returns approximately $15 \%$ of all Twitter activity $^{7}$. The trends/location stream provides a list of trending topics that is specific to a particular location. The United States

\footnotetext{
${ }^{7}$ http://dev.twitter.com/pages/streaming_api_concepts
} 
was used as the location for evaluation, as both experimental methods worked almost entirely with English tweets, and most of the trending topics from the United States were in English, leading to a more accurate basis for comparison than trending topics from the entire world. The streaming data was collected automatically using the cURL data transfer tool within a shell script. The streaming data was grouped into documents of equal duration. The first experiment used documents consisting of tweets collected over either ten minutes or one hour of streaming. The second experiment used documents consisting of tweets collected over fifteen minutes of streaming.

\section{B. Preprocessing}

The data was collected from the Twitter streaming API in JSON format and a parser to extract the tweets from the other information returned. Next the tweets were preprocessed to remove URL's, unicode characters, usernames, and punctuation. A dictionary containing approximately 180,000 common English words and acronyms was used to filter out tweets that did not contain at least $60 \%$ English words. Tweets were classified as spam and discarded if one word accounted for over half of the words in the tweet. After preprocessing, tweets were stored in two ways - in a collection in which each valid tweet was left intact, and in a "bag of words" style dictionary consisting of a unigram and the frequency of the unigram in the document.

\section{Baseline Data}

Baseline data was computed from the Edinburgh Twitter Corpus, a collection of over 97 million tweets collected over three months in late 2009 and early 2010. The corpus was divided into 1212 sections corresponding to one hour's worth of tweets, consisting of two bag-of-words dictionarys for each section - one containing unigrams and one containing bigrams. For the first experiment, the resulting documents were used independently of one another. For the second experiment, the documents were compiled into a comprehensive dictionary of 805,025 words with term frequency, document frequency, and $\mathrm{tf}$-idf weights computed for each word. For the first experiment, a specified number of baseline documents was used to compute average normalized term frequency. For the second experiment, the dictionary was used to provide document frequencies for terms and for the generation of a stop word list.

\section{Stop Words}

For each experiment, a list of stop words was used as an additional filter after preprocessing. A stop word is defined as a word that contains no meaning or relevance in and of itself, or a word that adds to the relevance of a result to a query no more often than would a random word [18].

For the first experiment, stop word were identified using a "lossy counting" algorithm [9]. The lossy counting algorithm identified the most frequent words in each of the 1212 baseline documents. All words that appeared as the most frequent in at least $75 \%$ of the baseline documents were classified as stop words. If a word in the test data was identified as a stop word, it was immediately removed from consideration as a potential trending topic.

For the second experiment, a word was considered to be a stop word if it matched one or more of the following criteria:

- If the word appeared in over 600 of the 1212 documents

- If the word had a total frequency of at least 3000 throughout all 1212 documents

- If the word was classified grammatically as a preposition or a conjunction

- If the word was a derivative of a word that occurred in 1200 or more documents (i.e. "can" occurs in all 1212 documents, so "can't," "could," "couldn't," and "could've" are also classified as stop words)

As with the first experiment, if a word in the test data was identified as a stop word, it was immediately removed from consideration as a potential trending topic.

\section{E. Selection Criteria}

For the first experiment, a combination of raw frequency and relative normalized term frequency was used. The raw frequency was used as a threshold, eliminating all terms that did not occur an average of at least one time for every minute of data collection. Normalized term frequency and average normalized term frequency was calculated for each remaining term, and the terms with the highest trending scores were identified as trending topics. Analysis was performed for both unigrams and bigrams. Entropy was also calculated for both unigrams and bigrams, but was not used as a selection criterion for this experiment.

The second experiment utilized a combination of raw frequency, tf-idf weighting, and entropy to identify trending topics. Once again, the raw frequency was used as a threshold, eliminating all terms that did not occur an average of at least one time for every minute of data collection. Term frequencyinverse document frequency weights were calculated for the remaining terms. Of the remaining terms, those with a tfidf weight below a threshold value (set at five greater than the length of data collection in minutes) were removed from consideration. Finally, terms with an entropy of less than 3.0 were removed, and the remaining terms were identified as trending topics.

\section{Vi. Evaluation Measures}

The first experiment was evaluated using precision, recall, and F-measure scores in comparison to the trending topics identified by Twitter. All three measures require calculating the number of true positives - that is, the items that were identified as trending topics both by the experimental method and Twitter's method. In addition, determining precision requires calculating the number of false positives - the items identified as trending topics by the experimental method that were not identified as trending topics by Twitter, and determining recall requires calculating the number of false negatives - the items identified as trending topics by Twitter that were not identified 


\begin{tabular}{|c|r|r|r|c|c|c|}
\hline Data Set & TP & FP & FN & Precision & Recall & F-measure \\
\hline Hourly Unigrams 1 & 8 & 22 & 24 & 0.2667 & 0.2500 & 0.2581 \\
\hline Hourly Unigrams 2 & 9 & 21 & 20 & 0.3000 & 0.3103 & 0.3051 \\
\hline Hourly Unigrams 3 & 4 & 26 & 27 & 0.1333 & 0.1290 & 0.1311 \\
\hline Hourly Unigrams 4 & 7 & 23 & 25 & 0.2333 & 0.2188 & 0.2258 \\
\hline Hourly Unigrams 5 & 6 & 24 & 22 & 0.2000 & 0.2143 & 0.2069 \\
\hline Hourly Unigrams 6 & 5 & 25 & 23 & 0.1667 & 0.1786 & 0.1724 \\
\hline Average & & & & 0.2167 & 0.2168 & 0.2167 \\
\hline Hourly Bigrams 1 & 4 & 26 & 7 & 0.1333 & 0.3636 & 0.1951 \\
\hline Hourly Bigrams 2 & 7 & 7 & 5 & 0.5000 & 0.5833 & 0.5385 \\
\hline Hourly Bigrams 3 & 3 & 27 & 6 & 0.1000 & 0.3333 & 0.1538 \\
\hline Hourly Bigrams 4 & 4 & 17 & 9 & 0.1905 & 0.3077 & 0.2353 \\
\hline Hourly Bigrams 5 & 2 & 28 & 7 & 0.0667 & 0.2222 & 0.1026 \\
\hline Hourly Bigrams 6 & 4 & 16 & 7 & 0.2000 & 0.3636 & 0.2581 \\
\hline Average & & & & 0.1984 & 0.3623 & 0.2564 \\
\hline 10 Minute Unigrams 1 & 13 & 17 & 19 & 0.4333 & 0.4063 & 0.4194 \\
\hline 10 Minute Unigrams 2 & 8 & 22 & 25 & 0.2667 & 0.2424 & 0.2540 \\
\hline 10 Minute Unigrams 3 & 12 & 18 & 19 & 0.4000 & 0.3871 & 0.3934 \\
\hline 10 Minute Unigrams 4 & 11 & 19 & 22 & 0.3667 & 0.3333 & 0.3492 \\
\hline 10 Minute Unigrams 5 & 8 & 22 & 24 & 0.2667 & 0.2500 & 0.2581 \\
\hline 10 Minute Unigrams 6 & 9 & 21 & 23 & 0.3000 & 0.2813 & 0.2903 \\
\hline Average & & & 0.3389 & 0.3167 & 0.3274 \\
\hline 10 Minute Bigrams 1 & 2 & 21 & 7 & 0.0870 & 0.2222 & 0.1250 \\
\hline 10 Minute Bigrams 2 & 1 & 23 & 8 & 0.0417 & 0.1111 & 0.0606 \\
\hline 10 Minute Bigrams 3 & 2 & 9 & 8 & 0.1818 & 0.2000 & 0.1905 \\
\hline 10 Minute Bigrams 4 & 3 & 9 & 8 & 0.2500 & 0.2727 & 0.2609 \\
\hline 10 Minute Bigrams 5 & 1 & 17 & 8 & 0.0556 & 0.1111 & 0.0741 \\
\hline 10 Minute Bigrams 6 & 2 & 16 & 8 & 0.1111 & 0.2000 & 0.1429 \\
\hline Average & & & & 0.1212 & 0.1862 & 0.1468 \\
\hline
\end{tabular}

Fig. 3: Table of precision, recall, and F-measure scores for both unigrams and bigrams from analysis of data sets consisting of six one-hour segments and six ten-minute segments of tweets from the Twitter Streaming API.

as trending topics by the experimental method. Precision is defined as

$$
P=\frac{T P}{T P+F P}
$$

where $T P$ is the number of true positives and $F P$ is the number of false positives. Recall is defined as

$$
R=\frac{T P}{T P+F N}
$$

where $T P$ is the number of true positives and $F N$ is the number of false negatives. The F-measure is the harmonic mean of the precision and recall, defined as

$$
F=2 \cdot \frac{P \cdot R}{P+R}
$$

The second experiment was evaluated using recall and relevancy scores. Recall was calculated in comparison to the trending topics identified by Twitter using two different methods of identifying true positives and false negatives. The first method only identified as true positives terms that exactly matched terms identified by Twitter as trending topics. Since the second experiment returned only bigrams, terms identified by Twitter as trending topics that were not identified by the experimental method were only considered to be false negatives if they were unigrams. The second method identified a term as a true positive if it either exactly matched a term identifed by Twitter as a trending topic or if it matched one part of a multigram trending topic. Any term identified as a trending topic by Twitter that was not identified as a trending topic by the experimental method was classified as a false negative. Relevance was calculated based on the evaluations of human volunteers. Volunteers were given a list of terms identified as trending topics and marked those that they felt were valid or relevant topics. The list contained both terms identified as trending topics by the experimental method and terms identified as trending topics by Twitter as a control. Relevance was calculated in the same manner as precision was calculated in the first experiment.
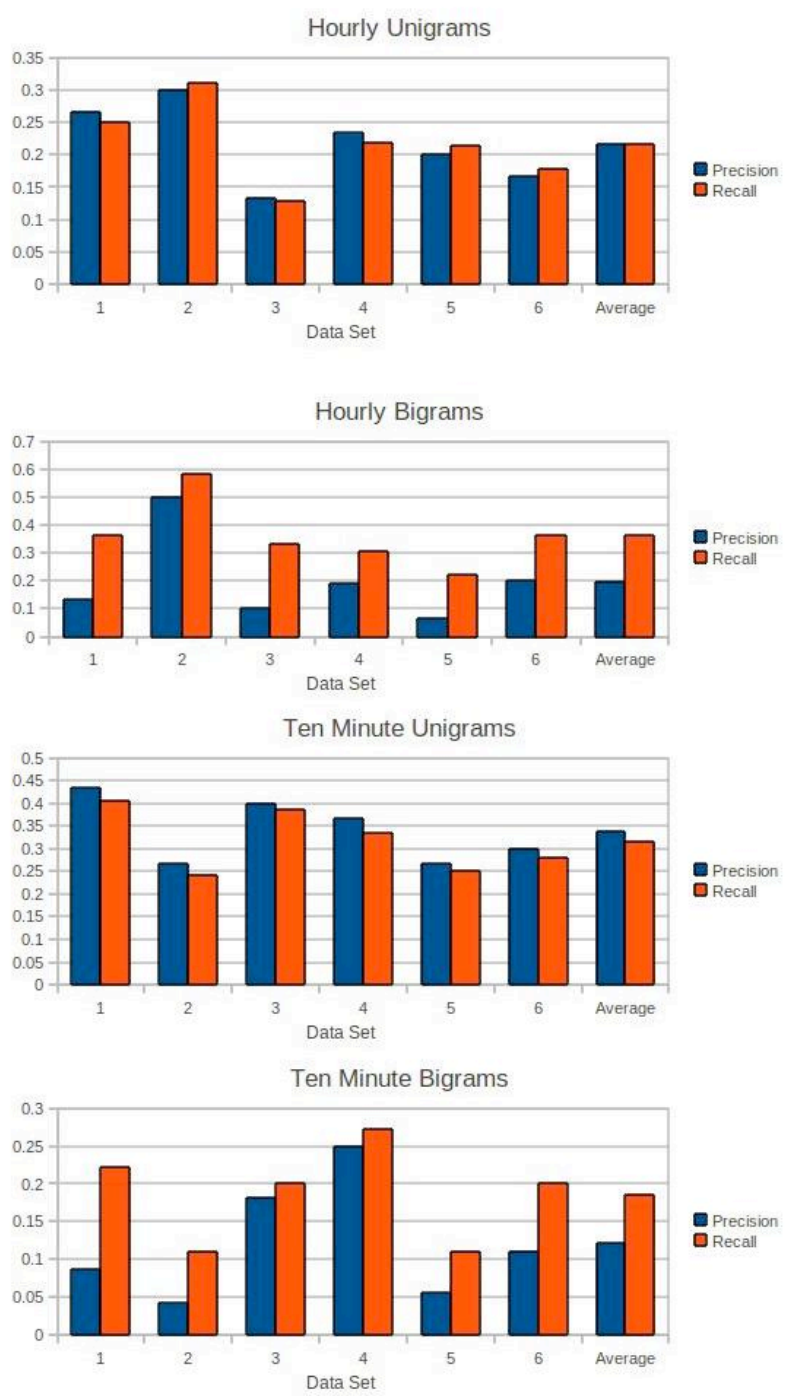

Fig. 4: Graph of precision and recall scores for both unigrams and bigrams from analysis of data sets consisting of six onehour segments and six ten-minute segments of tweets from the Twitter Streaming API.

\section{RESULTS}

For the first experiment, the hourly data sets had an average precision of 0.2167 and 0.1984 and an average recall of 0.2168 and 0.3623 for an F-measure of 0.2167 and 0.2564 for unigrams and bigrams, respectively. The ten minute data sets had an average precision of 0.3389 and 0.1212 and an average recall of 0.3167 and 0.1862 for an F-measure of 0.3274 and 0.1468 for unigrams and bigrams, respectively. A table of the 
results of the first experiment can be seen in Fig. 3 and a graph showing the precision and recall scores for each data set is shown in Fig. 4. Initial goals for this experiment were a precision score of at least 0.50 and a recall score of at least 0.75 , for an f-measure of at least 0.60 . The initial results are well below this, but within reasonable range the results of similar work, which produced f-measures in the range of 0.30 to 0.60 [1], [20].

For the second experiment, initial results gave an average precision of 0.2780 and an average recall of 0.7667 for an F-measure of 0.3988 as calculated by the first method of evaluation, and an average precision of 0.4075 and an average recall of 0.5985 for an F-measure of 0.4794 as calculated by the second method of evaluation. The initial results were evaluated by human volunteers as containing relevant topics $72.43 \%$ of the time, compared to $77.14 \%$ of the time for the terms identified by Twitter as trending topics. Substituting relevance scores for precision scores produces an F-measure of 0.7508 as evaluated by the first method of evaluation and and F-measure of 0.66 as evaluated by the second method of evaluation Given that the success criteria were a recall of 0.50 when evaluated with the terms identified by Twitter and a relevance of at least $75 \%$ that of the terms identified by Twitter, the data from the second experiment meets the conditions of success.

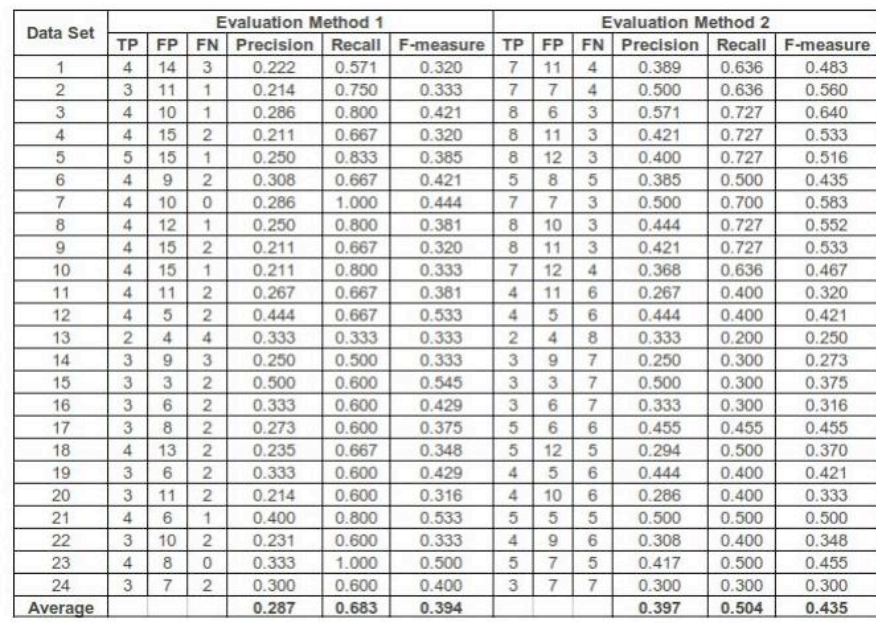

Fig. 5: Table of precision, recall, and F-measure scores for both unigrams from analysis of data sets consisting of 24 fifteen minute segments of tweets from the Twitter Streaming API.

\section{IMPROVEMENTS AND EXTENSIONS}

Based on the initial performance of the proposed method, there are several possible extensions and improvements for this project. One potential extention would be to expand the functionality of the unigram and bigram algorithms to identify trigrams or higher order n-grams as trending topics, instead of single words or bigrams. Other possible extensions of this project include interfacing with the Inouye project [6] and the Kaufmann project [7] in order to not only identify but summarize trending topics and normalize the syntax of the summaries, or adapting the method to be used as a predictive
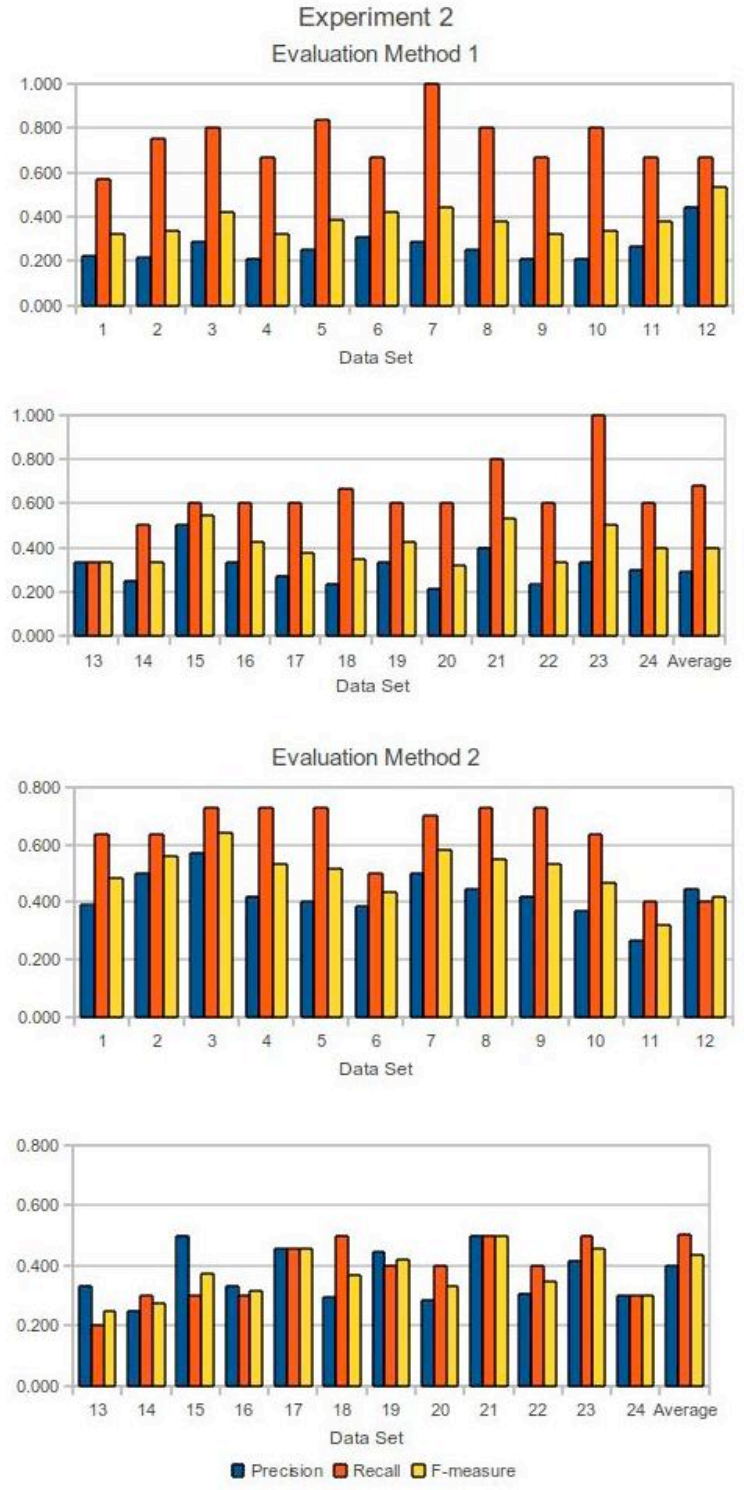

Fig. 6: Graph of precision, recall, and F-measure scores for both unigrams from analysis of data sets consisting of 24 fifteen minute segments of tweets from the Twitter Streaming API.

tool. One final extension could be in the evaluation process. Terms identified as trending topics could be compared not only to topics identified by Twitter as trending, but to topics identified as trending by other sources, such as Yahoo! $!^{8}$ or Google Trends ${ }^{9}$. Ideally, there will be time for at least two extensions to be implemented during the remainder of the time allotted for this project.

\section{CONCLUSION}

In this paper, we have outlined methodologies for using streaming data, tf-idf term weighting, normalized term frequency analysis, and other criteria to identify trending topics

\footnotetext{
${ }^{8} \mathrm{http} / / / \mathrm{www} . y a h o o . c o m$

${ }^{9} \mathrm{http}: / /$ www.google.com/trends
} 
on Twitter. The methods implemented detected and identified both unigrams and bigrams as trending topics. Preliminary results for the first experiment fell significantly short of the original goals, but were reasonably close to results produced by other approaches. Preliminary results for the second experiment seem to meet the success conditions put forth in this paper. The current state of the project allows room for extensions in the form of interfacing with other projects applying natural language processing techniques to Twitter. Finally, once all results are analyzed, this project hopefully will have demonstrated the ability of natural language processing tools to extract and identify pertinent information from a continuously changing corpus with an unconventional structure.

\section{REFERENCES}

[1] J. Allan, R. Papka, and V. Lavrenko, "On-line New Event Detection and Tracking," In Proceedings of ACM SIGR, pp. 37-45, 1998.

[2] M. Cheong, V. Lee, "Integrating Web-based Intelligence Retrieval and Decision-making from the Twitter Trends Knowledge Base," In Proceedings of CIKM 2009 Co-Located Workshops: SWSM 2009, pp. 1-8, 2009.

[3] N. Glance, M. Hurst, and T. Tomokiyo, "Blogpulse: Automated Trend Discovery for Weblogs," In WWW 2004 Workshop on the Weblogging Ecosystem: Aggregation, Analysis, and Dynamics, 2004.

[4] D. Gruhl, R. Guha, D, Liben-Nowell, and A. Tomkins, "Information Diffusion Through Blogspace," In Proceedings of the 13th International Conference on the World Wide Web, pp.491-501, 2004.

[5] D. Hiemstra, "A probabilistic justification for using tf $\times$ idf term weighting in information retrieval," International Journal on Digital Libraries, vol. 3, no. 2, pp. 131-139, 2000.

[6] D. Inouye, "Multiple Sentence Microblog Summarization," In REU Site for Artificial Intelligence, Natural Language Processing and Information Retrieval Research Projects, 2010. Forthcoming

[7] J. M. Kaufmann, "Syntactic Normalization of Twitter Messages," In REU Site for Artificial Intelligence, Natural Language Processing and Information Retrieval Research Projects, 2010. Forthcoming

[8] K. Kireyev, L. Palen, K. Anderson, "Applications of Topics Models to Analysis of Disaster-Related Twitter Data," In NIPS Workshop on Applications for Topic Models: Text and Beyond, 2009.

[9] G. Manku and R. Motwani, "Approximate Frequency Counts Over Data Streams," In Proceedings of the 28th VLDB Conference, Hong Kong, China, 2002.

[10] R. Nallapati, A. Feng, F. Peng, and J. Allan, "Event Threading within News Topics," In Proceedings of the Thirteenth ACM Conference on Information and knowledge management, pp.446-453, 2004.

[11] R. Perera, S. Anand, P. Subbalakshmi, and R. Chandramouli, "Twitter Analytics: Architecture, Tools and Analysis."

[12] S. Petrovic, M. Osborne, and V. Lavrenko, "Streaming First Story Detection with appilcation to Twitter," In Proceedings of NAACL, 2010

[13] S. Petrovic, M. Osborne, and V. Lavrenko, "The Edinburgh Twitter Corpus," In Proceedings of NAACL Workshop on Social Media, 2010.

[14] T. Sakaki, M. Okazaki, and Y. Matsuo, "Earthquake Shakes Twitter Users: Real-time Event Detection by Social Sensors," In WWW2010, 2010.

[15] G. Salton and C. Buckley, "Term-Weighting Approaches in Automatic Text Retrieval," Information Processing and Management, vol. 24, no. 5, pp. 513-523, 1988.

[16] B. Shaparenko, R. Caruana, J. Gehrke, and T. Joachims, "Identifying Temporal Patterns and Key Players in Document Collections," In Proceedings of the IEEE ICDM Workshop on Temporal Data Mining: Algorithms, Theory, and Applications (TDM-05), pp.165-174, 2005.

[17] B. Sharifi, M. Hutton, and J. Kalita, "Experiments in Microblog Summarization," In NAACL-HLT 2010, Los Angeles, 2010.

[18] W. J. Wilbur and K. Sirotkin, "The Automatic Identification of Stop Words," Journal of Information Science, vol. 18, pp. 45-55, 1991.

[19] W. J. Wilbur and Y. Yang, "An Analysis of Statistical Term Strength and its Use in the Indexing and Retrieval of Molecular Biology Texts," Computers in Biology and Medicine,vol. 26, no. 3, pp. 209-22, 1996.

[20] Y. Yang, T. Pierce, J. Corbonell, "A Study on Retrospective and On-Line Event Detection," In Proceedings of the 21st ACM SIGR, 1998. 